$\mathbb{T}$ periodica polytechnica

Mechanical Engineering

$52 / 2$ (2008) 7781

doi: 10.3311/pp.me.2008-2.07

web: http://www.pp.bme.hu/me

(c) Periodica Polytechnica 2008

RESEARCH ARTICLE

\section{Human wavelength identification, numerical analysis and statistical evaluation}

Balázs Vince Nagy / Zoltán Németh / György Ábrahám

Received 2008-10-17

\begin{abstract}
Monochromatic wavelength identification never had such significance as today when several lighting applications favor the use of light emitting diodes as light sources. People with normal color vision designing the color appearance of a monochromatic lighting solution usually do not consider the different appearance of these stimuli for color blind. In our study we have measured the monochromatic color identification of more than 100 observers with different types of color vision and we have found significant differences in their color identification. The results should be implemented in all illumination design where the use of LEDs is considered.
\end{abstract}

\section{Keywords}

Human color vision measurement $\cdot$ color identification $\cdot$ color vision deficiency.

\section{Balázs Vince Nagy}

Zoltán Németh

Department of Mechatronics, Optics and Engineering Informatics, H-1521 Budapest,, Hungary

\section{György Ábrahám}

Department of Mechatronics, Optics and Engineering Informatics, H-1521 Budapest, Hungary

\section{Introduction}

Human color vision has been studied broadly during the last two centuries $[8-10,15]$. Today we already have knowledge on how color blindness is caused by photo pigment spectral sensitivity changes in the human photodetectors (Nathans et al., 1986) [13] and we can distribute the color deficiency types into several categories [1].

Color identification or more popularly known as color naming has been also studied by the scientific community. Although their interest was more in the linguistic or geographic distribution of color terms [3]; [11, 14] or in their characterization in color systems [6,7] rather than in the relation of color names to spectral features [5] or color deficiency [16].

Therefore it seems to be a righteous aim to research the spectral background of color identification in human color vision with special focus on the abilities of the color deficient.

\section{Methods}

Our research group has conducted a test series with a special instrument built for the monochromatic measurement of color vision. The so called PDT instrument (where the name PDT comes from the abbreviation of the three photoreceptors in the human eye, namely Protos, Deuteros and Tritos - Fig. 11 was designed to provide quasi monochromatic $(\mathrm{FWHM} \approx 10 \mathrm{~nm}) 4^{\circ}$ stimuli (7-14) through an ocular (5) with a $10^{\circ}$ white background (1-13) to avoid color adaptation. The background color can be changed for different measurement purposes with the help of a filter wheel (3). In our tests we used the stimulus light of a conventional incandescent bulb (7) going through an interference filter (IF, 9) and combined with the white background light (1) by a beam splitter prism (4). The PC controlled IF filter was tuned continuously in the $380 \mathrm{~nm}-730 \mathrm{~nm}$ spectral range.

During our tests we have used the PDT instrument to measure 31 subjects with normal color vision [2] and to compare the results to the data of 100 color deficient subjects. Color deficiency was tested by means of Ishihara plates, Farnsworth 100 Hue test and Heidelberg anomaloscope to provide diagnosis on the type and severity (Table 1) [4, 12].

During the tests the subjects looked into the ocular of the in- 


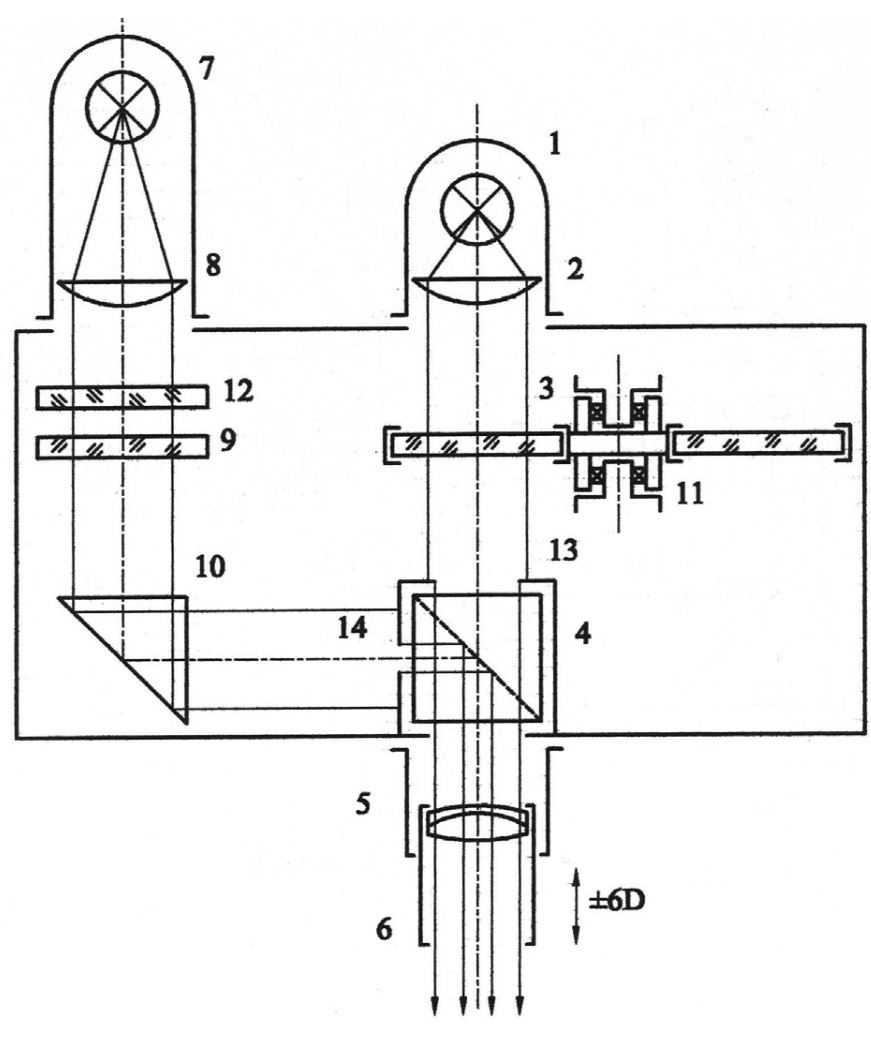

Fig. 1. Optical scheme of the test instrument (PDT)

Tab. 1. Subject categories. Protanomals and deuteranomals have defected red and green sensitive photoreceptor, respectively. ' $\mathrm{l}$ ' and ' $\mathrm{m}$ ' indicate protos (long wavelength sensitive) defect and deuteros (medium wavelength sensitive) defect respectively while 10-25 indicate increasing severity.

\begin{tabular}{lccccccccc}
\hline \multirow{2}{*}{ Type } & \multirow{2}{*}{ Normal } & \multicolumn{4}{c}{ Protanomals } & \multicolumn{4}{c}{ Deuteranomals } \\
\cline { 3 - 10 } & & $\mathrm{I} 10$ & $\mathrm{I} 15$ & $\mathrm{I} 20$ & $\mathrm{I} 25$ & $\mathrm{~m} 10$ & $\mathrm{~m} 15$ & $\mathrm{~m} 20$ & $\mathrm{~m} 25$ \\
\hline No. & 31 & 9 & 9 & 8 & 19 & 17 & 11 & 10 & 17 \\
\hline
\end{tabular}

strument and had the task to name any colors that they see using color categories such as purple, blue, turquoise, green, yellow, orange, red, white and n/a. Subjects could use more terms to describe one stimulus which was especially necessary among the color deficient who had more confusion about the color naming than normals.

The instrument was controlled randomly by the PC so that the subjects did not see the colors in sequence. Measurements were repeated three times each. Test subjects were $100 \%$ male in the color deficient group and 71\%-23\% males and females respectively among the normals (ages were between 17 and 55).

\section{Results}

Figs. 2a, b show the normalized results of cumulated measurements for people with normal color vision and for extreme protanomals, respectively. The figures show the relative color terms used at each wavelength demonstrating how the subjects identify the main colors in the wavelength range.

Color terms are indicated as purple, blue, turquoise, green, yellow, orange, red from left to right in Fig. 2a. Fig. 2p shows extreme red color deficients according to the characteristics of people with extreme protanomaly. They loose their clear color identification ability mostly towards the higher wavelength end of the spectrum. The severity of deficiency increases with the confusion in naming colors especially towards the green-red range of the spectrum. We aimed to characterize this confusion and introduced a numerical value that can distinguish the identification of different types of color deficient.

\section{Numerical characterization of color identification}

As the results of measurements are only given verbally with the color terms, in our evaluation we assigned numerical values to each color name used in Table 2

Tab. 2. Numerical values (scores) assigned to the color terms

\begin{tabular}{cccccccc}
\hline Color term & Purple & Blue & Turquoise & Green & Yellow & Orange & Red \\
\hline Value & 100 & 110 & 120 & 130 & 140 & 150 & 160 \\
\hline
\end{tabular}

With these values every single measurement could have been characterized numerically and we could display the color naming dependency of the individuals along the wavelength axis. Moreover the results of the groups could have been compared with mathematical statistical approaches.

The following figures show the distribution of numerically defined color names versus wavelength for the different color deficient types together with the distribution for normals.

The distributions clearly show the higher alterations from the normals as severity increases. At the protanomals the decreasing 'red-end' is also clearly displayed (Fig. 3 b-e). This is a specific feature of protanomal color vision that they lack sensitivity at the higher wavelength end of the spectrum. Protanomal types also show high standard deviation in color naming especially at the higher wavelength range. This also can be explained with the loss of sensitivity thus more significant confusion in characterizing the stimuli.

If we compare the distribution of color terms the color deficient types show deviation from the normal distribution. This difference can be analyzed mathematically using the assigned color name values instead of the color terms. The distributions differ so that their centroid wavelengths are shifted to lower wavelength ranges. Thus calculating the centroid wavelength for each individual measurement we can obtain a single numerical value to characterize the monochromatic color identification ability of the subject. The calculation of the centroid is done according to Eq. (1):

$$
\frac{\int_{400}^{\lambda_{c}} I D(\lambda)}{\int_{400}^{700} I D(\lambda)}=0.5,
$$

where $\operatorname{ID}(\lambda)$ is the specific numerical value assigned to the color term used by a given wavelength and $\lambda_{c}$ is the centroid wavelength. 

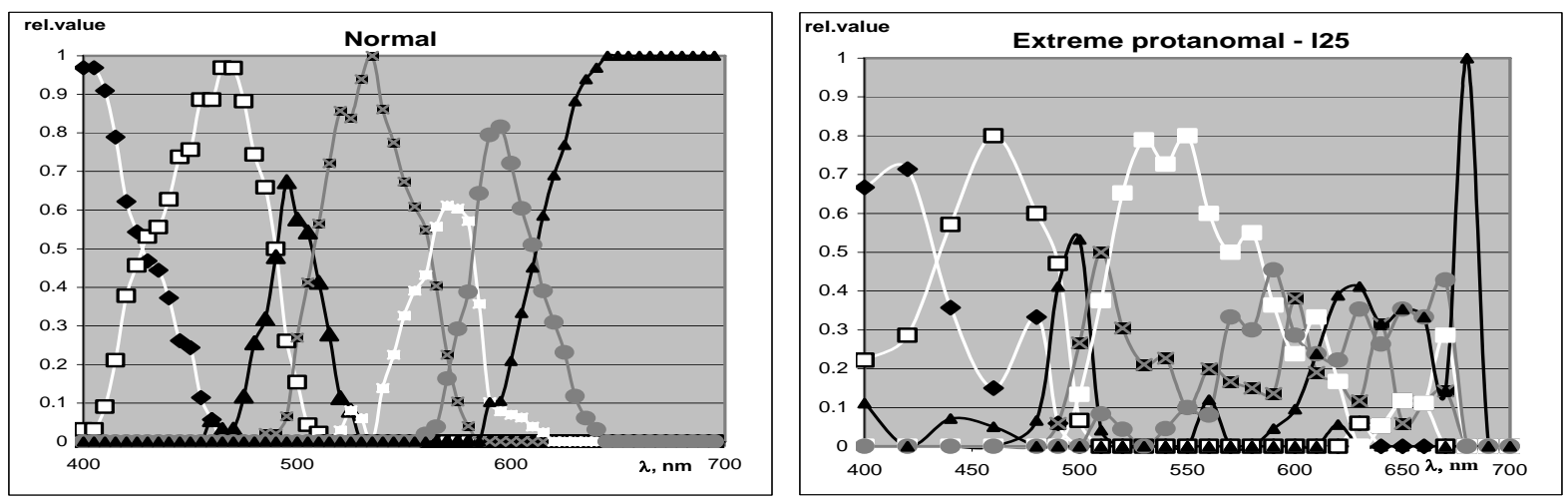

Fig. 2. Color naming distribution along the wavelength for people with normal color vision (2a) and for extreme protanomals (2b).

The equation was calculated with a well fitting $(\mathrm{R}>0,999)$ $5^{\text {th }}$ grade polynomial regression onto the normalized color score distribution of each measurement result. This way the centroid wavelengths of all groups could have been determined (Table 3 ).

Tab. 3. Average centroid wavelengths of the color vision types.

\begin{tabular}{lll}
\hline & & $\lambda_{c}, \mathbf{n m}$ \\
\hline Normal & & $\mathbf{5 6 6 . 0 5 1} \pm 0.691$ \\
\hline \multirow{3}{*}{ Protanomal } & $\mid 10$ & $\mathbf{5 6 6 . 2 3 3} \pm 0.409$ \\
& $\mid 15$ & $\mathbf{5 6 5 . 4 3 3} \pm 0.770$ \\
& $\mathrm{I} 20$ & $\mathbf{5 6 4 . 4 4 5} \pm 1.037$ \\
& $\mathrm{I} 25$ & $\mathbf{5 6 2 . 9 3 8} \pm 1.798$ \\
\hline \multirow{3}{*}{ Deuteranomal } & $\mathrm{m} 10$ & $\mathbf{5 6 6 . 2 3 6} \pm 0.946$ \\
& $\mathrm{~m} 15$ & $\mathbf{5 6 5 . 6 6} \pm 0.450$ \\
& $\mathrm{~m} 20$ & $\mathbf{5 6 5 . 1 0 3} \pm 0.910$ \\
& $\mathrm{~m} 25$ & $\mathbf{5 6 0 . 4 6 8} \pm 1.376$ \\
\hline
\end{tabular}

Although the differences between the centroids of the different color vision types are relatively small they also have quite small standard deviations. The significance in the differences between the centroids can be characterized with mathematical statistical methods.

To be able to apply strong mean comparison tests (such as the T-test) we verified the normality of the data distribution in each category. Using both Kolmogorov-Smirnov and Shapiro-Wilks normality tests we can conclude that neither distribution differs significantly from normal distribution. This allows us to apply T-test for the mean comparison whose result is considered to be stronger than the ones for non-normal distributions.

The T-test was applied to compare all results of the neighboring groups using the SPSS statistical analysis software pack. Table 4 shows the groups compared and the significance level of difference between them.

The results show high significance levels for almost all neighboring groups except for the normals compared to slight deficient cases. This means that most of the neighboring groups have a high probability not to be from the same distribution thus practically can be considered different.

\section{Discussion}

The statistical analysis shows that although the differences are small between average centroid wavelengths of the deficiency types, there is usually a significant difference between them. Normal and slight anomal identifications do not differ significantly which agrees quite well with the experiences of our research group that slight color deficient people have very similar color naming abilities as normals have [2, 16]. That is why these groups cannot be differentiated from normals with only color identification testing but they need also color discrimination checks such as Ishihara plates. The differences between protanomals and deuteranomals are also not significant but these main types can easily be distinguished by analyzing the red end results of the spectrum, namely if such reduced red end sensitivity exists they shall be protanomals. However the lack of red end sensitivity is significant only with a defined background adaptation. Tests on the PDT instrument show that all protanomal types have lack of sensitivity at 700nm using the instrument's white adaptation background. If monochromatic identification test is to be carried out on other instruments, the white background and the red stimuli with luminance contrast similar to that of the PDT should be applied. This is currently around 8.8\% at $700 \mathrm{~nm}$.

Based on the measurement and evaluation results we can establish a test algorithm for the color identification analysis of red-green color deficiency types (Fig. 4).

Following the method described in the block diagram we conclude to a diagnosis based on color identification test that could identify different color deficient types in the color naming distribution.

The results show that as color identification confusion increases with the severity of color deficiency at protanomal and deuteranomal types there is still a possibility to objectively characterize the differences between them. Therefore we can say that color naming ability has its specific characteristics at the examined types of color deficiency. 

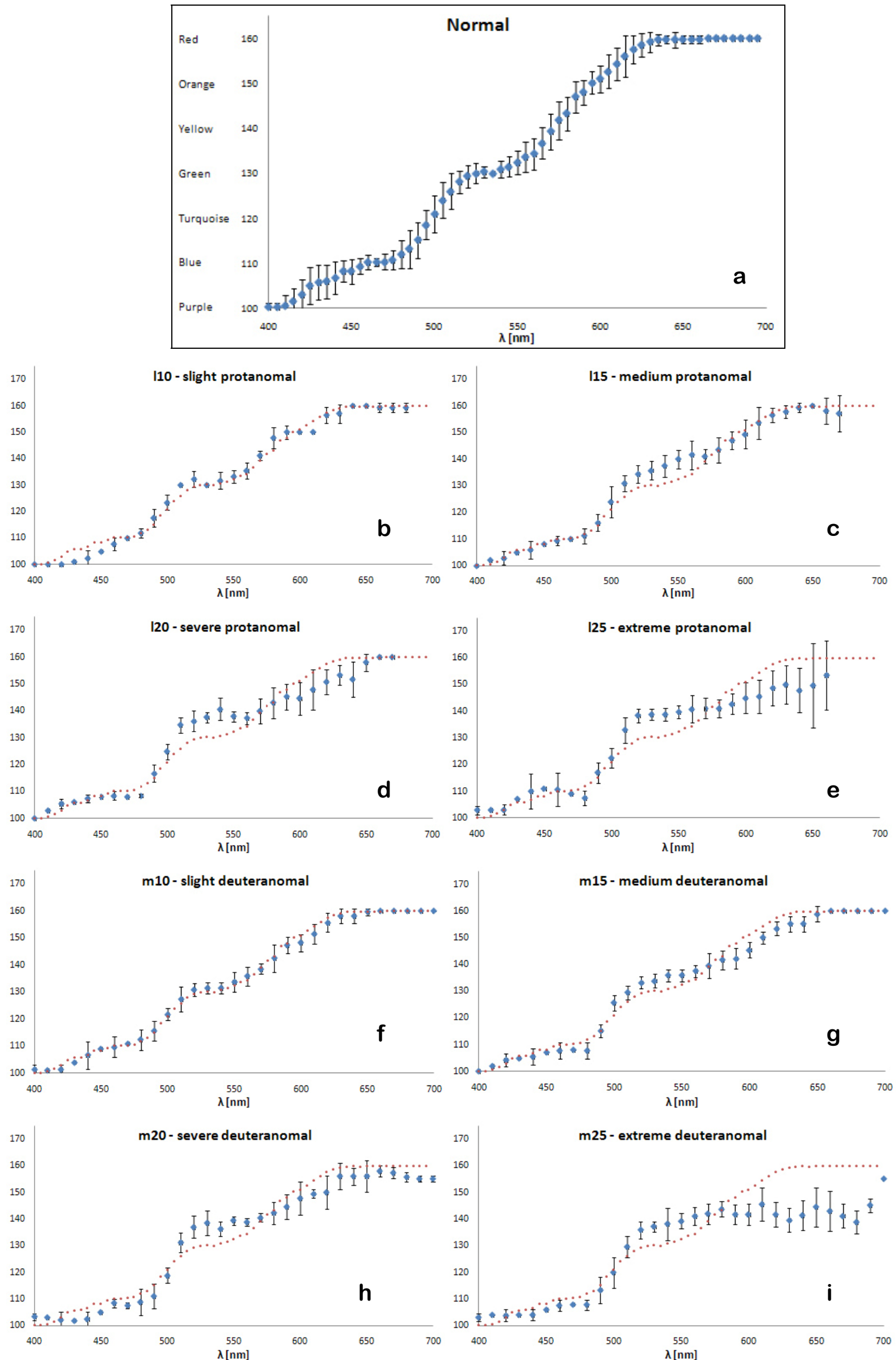

Fig. 3. Distribution of numerically defined color names for the different color deficient types together with the distribution of normals (smaller dots).

Fig.3a shows the results for normals, Fig.3b-e for protanomals and Fig.3f-i for deuteranomal types with increasing severity. 
Tab. 4. Statistical differences between deficient types

\begin{tabular}{cccccccccc}
\hline Group 1 & Normal & $\mid 10$ & Normal & I15 & I20 & Normal & m10 & m15 & m20 \\
\hline Group 2 & $\mid 10$ & $\mid 15$ & $\mid 15$ & $\mid 20$ & $\mid 25$ & m10 & m15 & m20 & m25 \\
\hline Significance level, \% & 23.3 & 59.4 & 96.6 & $\mathbf{9 7 . 3}$ & $\mathbf{9 8 . 8}$ & 51.5 & 95.9 & $\mathbf{8 9 . 6}$ & $\mathbf{9 9 . 9}$ \\
\hline
\end{tabular}

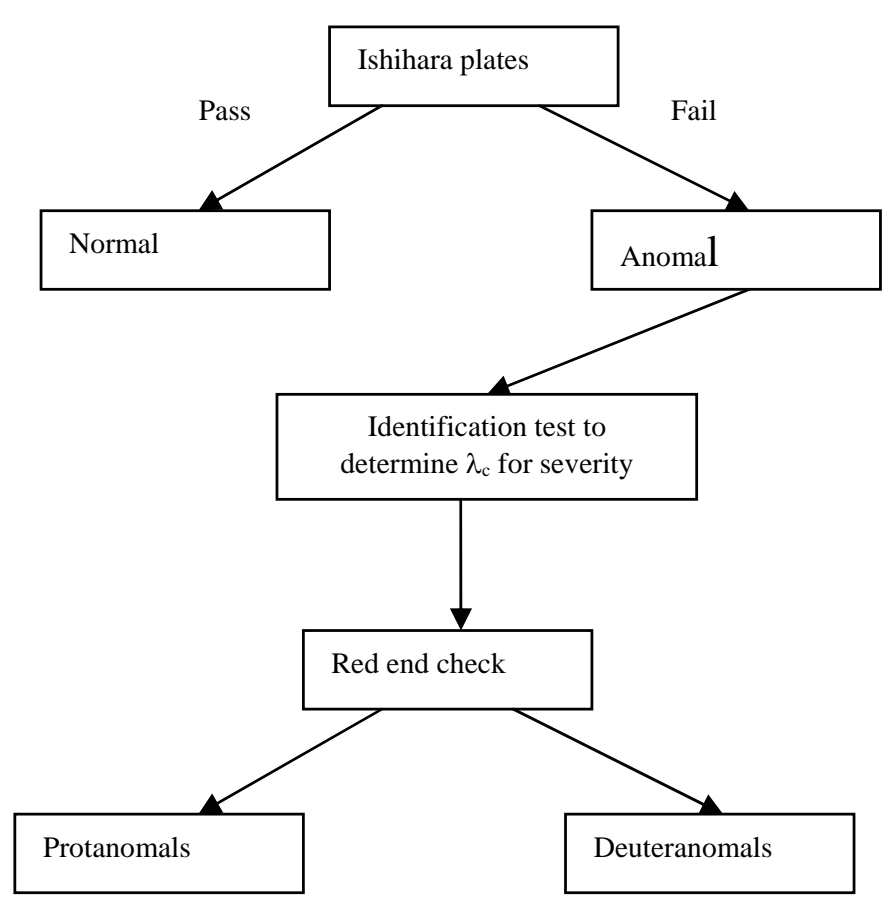

Fig. 4. Color identification test algorithm

\section{References}

1 Ábrahám Gy, Szappanos J, Wenzel K, Method and optical means for improving or modifying colour vision and method for making said optical means, 1993. USA Patent No. 5774202.

2 Ábrahám Gy, Nagy B V, Colour identification based on opponent colour signals, Proc. of Temporal and Spatial Aspects of Light and Colour Perception and Measurement, Veszprém. CIE x025:2003, 2003, pp. 123-126.

3 Berlin B, Kay P, Basic color terms: their universality and evolution, Berkeley: University of California Press, 1969.

4 Birch J, Diagnosis of Defective Colour Vision, Oxford University Press, 1993.

5 Byrne A, Hilber D, Color realism and color science. Behavioral and Brain Sciences, Vol. 26, Cambridge University Press, 2003.

6 Franklin A, Clifford A, Williamson E, Davies I, Color term knowledge does not affect categorical perception of color in toddlers, J. Experimental Child Psychology 90 (2005), 114-141, DOI 10.1016/j.jecp.2004.10.001.

7 Guest S, D. van Laar, The structure of colour naming space, Vision Research 40 (2000), 723-734, DOI 10.1016/S0042-6989(99)00221-7.

8 Helmholtz H L F von, Über die Theorie der zusammengesetzten Farben, Annales de Physique 887 (1852), 45-66.

9 Hering E, Outline of a Theory of the Light Sense (Hurvich L, Jameson D, eds.), Harvard University Press, Cambridge, MA., 1964, 1905.

10 Hurvich L M, Jameson D, An opponent process theory of color vision, Psychological Review (1957), 384-404.

11 Kay P, Regier T, Resolving the question of color naming universals $\mathbf{1 0 0}$ (2003), no. 15, DOI 10.1073/pnas.1532837100. This contribution is part of the special series of Inaugural Articles by members of the National Academy of Sciences PNAS.
12 Nagel W A, Neue Erfahrungen über das Farbensehen der Dichromaten auf grossen Felde, Zeitschrift für Sinnesphysiologie 41 (1907), 319-337.

13 Nathans J, Piantanida T P, Eddy R L, Shows T B, Hogness D S, Molecular genetics of inherited variation in human color vision, Science $\mathbf{2 3 2}$ (1986), 203-210.

14 Rosch-Heider E R, Universals in color naming and memory, Journal of Experimental Psychology 93 (1972), 10-20.

15 Young T, The Bakerian lecture: On the theory of light and colours, Philosophical Transactions of the Royal Society, London, 1802, pp. 12-48.

16 Wenzel K, Kovács T, Colour identification on PDT 2000 instrument, National Physical Laboratory London, 1999. 